\title{
Coherence resonance versus synchronization in a periodically forced self-sustained system
}

\author{
A. G. Balanov, ${ }^{1}$ N. B. Janson, ${ }^{1}$ D. E. Postnov, ${ }^{2}$ and P. V. E. McClintock ${ }^{1}$ \\ ${ }^{1}$ Department of Physics, Lancaster University, Lancaster, LA1 4YB, United Kingdom \\ ${ }^{2}$ Department of Physics, Saratov State University, Astrakhanskaya 83, 410026, Saratov, Russia
}

(Received 6 June 2001; published 2 April 2002)

\begin{abstract}
A fundamental relationship between coherence resonance (CR) and phase synchronization in a self-sustained system in the presence of noise is addressed. A Van der Pol system synchronized by external forcing is taken as an example. It is shown that, in breaking down synchronization, applied noise creates a new ordered motion whose coherence depends resonantly on its intensity, i.e., CR occurs. The same is true for both types of synchronization, via phase locking and via suppression: only the mechanisms of CR differ. The result is valid for any order $n: m$ of synchronization.
\end{abstract}

DOI: 10.1103/PhysRevE.65.041105

PACS number(s): 05.40.Ca, 05.20.-y, 05.45.-a

Synchronization is a fundamental nonlinear phenomenon, manifesting itself in phase-locking or frequency suppression of oscillations in interacting or forced self-sustained systems [1]. It arises in diverse areas of science, including, e.g., chemistry, astronomy, and physiology [2]. The contemporary perception of synchronization in the presence of noise is in many respects still based on the general theory developed by the late Stratonovich [3] for phase-locked periodic oscillators influenced by noise. Although created for periodic oscillations, the methods of the theory have also been usefully applied to studies of the synchronization of irregular motion [4]. It is generally accepted that noise wrecks phase coherence in synchronized systems, and thus disrupts synchronization. However, it is now well known that in nonlinear systems noise can often evoke a highly counterintuitive response by playing a creative role. For example, in Ref. [5] it was shown that external noise applied to a passive system can reduce the total noise at its output. A striking example is stochastic resonance, in which noise added to a nonlinear system enhances its response to a useful signal (see, e.g., Ref. [6], and references therein). Another phenomenon is known as coherence resonance (CR), where noise induces oscillations whose coherence depends resonantly on the noise intensity. It has been studied numerically [7-9] and recently confirmed by analog electronic experiment [10]. In particular, CR was demonstrated for an equation of form $\dot{x}$ $=a-\cos x+\xi(t)$, e.g., in Ref. [11]. Such an equation was earlier shown [3] to describe the phase difference of a forced periodic oscillator under the influence of noise for the simplest form of phase synchronization, namely, 1:1 phaselocking. Therefore, CR can be expected to manifest itself in the power spectra of a 1:1 phase-locked system. But what is the fundamental relationship between these two seemingly very different phenomena, i.e., phase synchronization in its most general form and CR? Is there any difference between the mechanisms via which noise causes the breakdown of synchronization for locking and suppression? How do the Fourier spectra evolve with noise intensity?

In the present paper we demonstrate that in the course of disrupting either kind of synchronization, i.e., locking or suppression, noise induces a new coherent motion marked by the appearance of an additional peak in the spectral density of oscillations. There is an optimal value of noise intensity that maximizes the height of this peak, and thus the regularity of the corresponding motion. It is therefore a form of CR. It arises for almost any point within the region of locking; whereas, in the region of suppression, it occurs only near the boundary. Due to the generality of the synchronization mechanisms our results are applicable to any $n: m$ resonance.

The harmonically forced Van der Pol oscillator has long been used as a paradigm for the exploration of synchronization phenomena. In the presence of noise it can be described by

$$
\begin{gathered}
\dot{x}=y, \\
\dot{y}=\varepsilon\left(1-x^{2}\right) y-\omega_{0}^{2} x+C \sin \Omega t+D \xi(t) .
\end{gathered}
$$

Here $\varepsilon=0.2$ is the nonlinearity parameter, $\omega_{0}=1.0$ and $\Omega$ are, respectively, the frequencies of self-oscillation and of external forcing, $C$ is the forcing strength, and $\xi$ represents Gaussian white noise of zero mean and unity variance whose intensity is $D$. In our study we used both numerical simulations and analogue electronic modelling techniques [12]. Figure 1 shows schematically the analog circuit used for modeling of Eq. (1) [13]. The voltages at points $A$ and $B$ correspond to the $x$ and $y$ coordinates of system (1), respectively.

The noiseless case $D=0$ has been discussed, e.g., in Ref. [1]. It was shown that, for a certain relationship between the amplitude $C$ and frequency $\Omega$ of the external forcing one can synchronize self-oscillations so that the frequencies of the system and of the external perturbation become related as

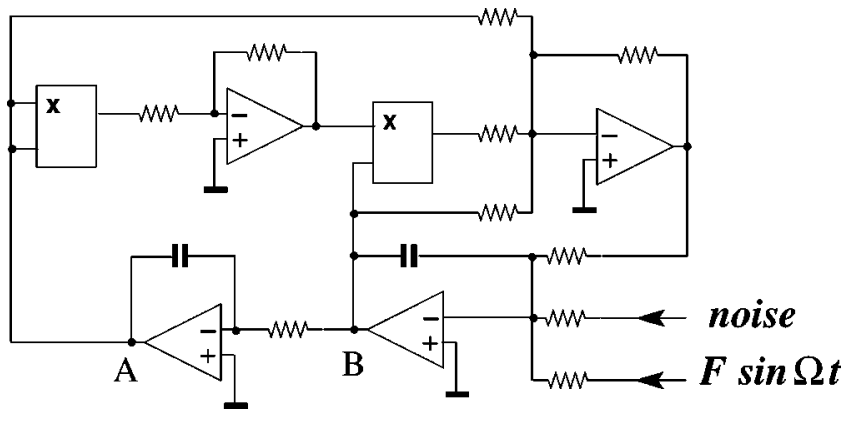

FIG. 1. Circuit (schematic) used for modeling (1). 


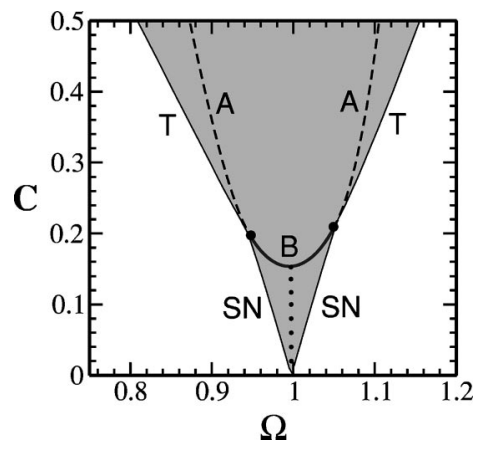

FIG. 2. Part of the 1:1 synchronization region (shaded) for the system (1). SN and $T$ label the saddle-node and torus birth/death bifurcation lines, respectively. In the area within the tongue below curve $B$ a resonant torus exists whose structure is shown in Fig. 5(a). In the region above curve $B$ only the stable cycle shown in Fig. 5(b) exists.

$n: m$, where $n$ and $m$ are integers. Thus, $n: m$ synchronization occurs. In the $(C, \Omega)$ parameter plane, the regions of synchronization form so-called Arnold tongues [14]. A portion of the 1:1 synchronization region for Eq. (1) is plotted in Fig. 2. There are two distinct synchronization mechanisms, namely, via phase locking and via suppression of natural oscillations, which are associated with two different bifurcations [1]. Curve $B$ divides the Arnold tongue into two parts in which the structure of the phase space is qualitatively different. The system can therefore be expected to respond differently to noise in different regions of the parameter plane.

We now present the results of experimental and numerical investigations of noise-induced effects in an initially synchronized system. First, consider the lower part of the Arnold tongue of Eq. (1) close to the synchronization boundary (Fig. 2 ). We set the external forcing parameters as $C=0.06, \Omega$ $=1.0118$ for $1: 1$ synchronization and $C=0.3, \Omega=0.33216$ for 1:3 synchronization. Figure 3 shows how the oscillation spectra evolve as the noise intensity is increased [15], as established by analogue electronic experiments. It is clearly evident that, in addition to periodic oscillations arising from resonant behavior between self-sustained oscillations and

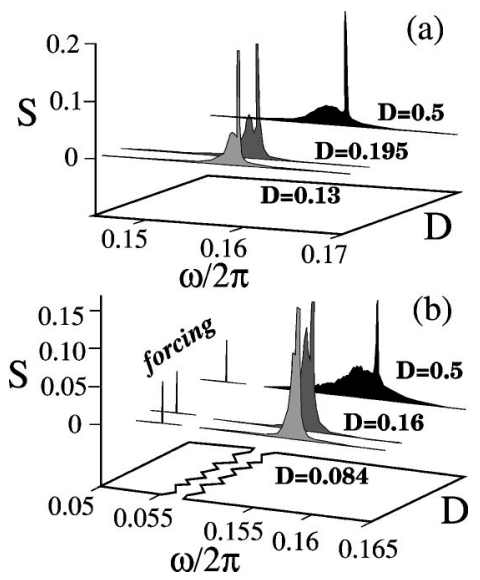

FIG. 3. Spectral density of oscillations versus noise intensity $D$ in the analogue experiment for (a) 1:1 synchronization, (b) 1:3 synchronization.

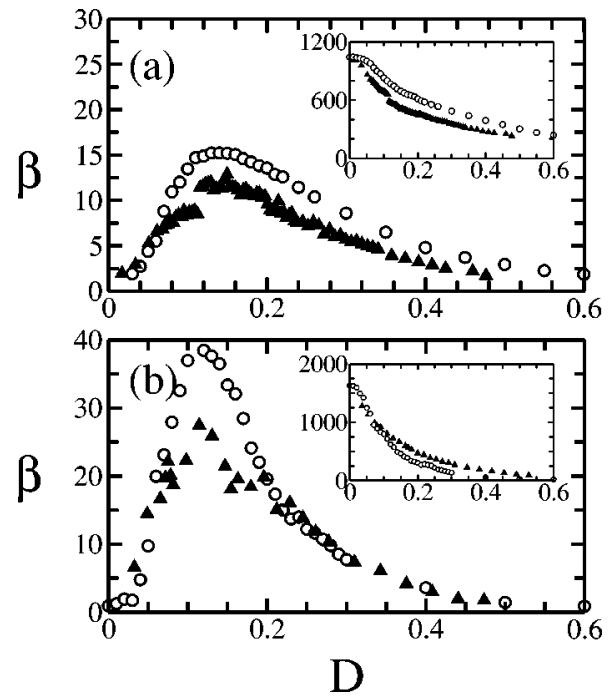

FIG. 4. Regularity $\beta$ of the noise-induced spectral peak as a function of noise intensity $D$ for synchronization via frequency locking for (a) $n: m=1: 1$ and (b) $n: m=1: 3$ from numerical simulation $(\bigcirc)$ and analog experiment $(\boldsymbol{\Delta})$. Inset: $\beta$ for the main peak as a function of noise intensity.

forcing, a new timescale is introduced by noise. It manifests itself through the appearance of a new spectral peak beside (here to the left of) the main one corresponding to synchronized oscillations. The position of the latter is the same as in the absence of noise and the mean frequency is the integral average of these two peaks.

Note that, unlike the case of stochastic resonance, noise does not enhance the system's response to the applied forcing. Instead, it gives rise to a new motion whose frequency differs from those of the already existing processes. The phenomenon is seen more clearly in Fig. 3(b) for 1:3 synchronization, where the three spectral peaks are well distinguished; whereas for 1:1 synchronization we can see only two peaks, because the self-oscillation peak coincides with that of forcing due to synchronization. Numerical simulations have yielded very similar results. The heights and widths of the noise-induced peaks change with variation of the noise intensity; the coherence or regularity of the new motion must presumably vary in a similar way.

To quantify the coherence or regularity of the oscillations corresponding to the new spectral peak we use a quantity that can be interpreted as a signal-to-noise ratio $\beta$ $=H \omega_{p} / \Delta \omega$, where $H$ is the height of the peak at the frequency $\omega_{p}$ and $\Delta \omega$ is the peak width at the height $H / 2$. Figure 4 displays the dependence of the new peak's regularity $\beta$ on noise intensity $D$ (a) for $1: 1$ and (b) for $1: 3$ frequency locking as determined by numerical simulation and analog experiment. In both graphs the coherence maxima are well defined, constituting evidence for coherence resonance. The numerical and analogue results are in satisfactory agreement. We can conclude therefore that, as the noise intensity in the initially synchronized system increases, the transition from the synchronous to the asynchronous regime involves passage through another coherent motion with an additional 

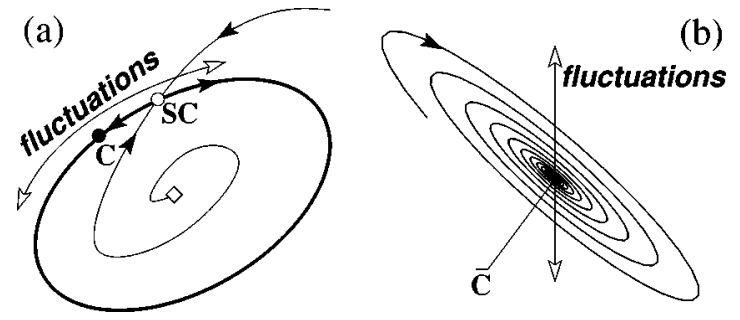

FIG. 5. Poincare sections of the harmonically forced selfsustained system for synchronization realized via (a) phasefrequency locking and (b) suppression. $C$ and SC denote the stable and saddle cycles, respectively.

timescale induced by the noise. The regularity $\beta$ of the main peak, corresponding to the initially synchronized motion decreases monotonically with noise intensity (see insets): so it seems that the proximity of the whole system's dynamics to being quasiperiodic is maximal when the regularity of noiseinduced motion is maximal, that is, for the optimal value of $D$. For small noise intensity the contribution from the new peak to the common motion is very small, and oscillations of the system are close to the unperturbed periodic dynamics, but for strong noise all coherence is destroyed. Similar spectral evolution was observed for other values of the parameters $C$ and $A$ within the synchronization tongue below line $B$ in Fig. 2.

We now consider how noise can generate a new coherent motion on the way to destroying synchronization. Consider first the lower part of the Arnold tongue. Here, a resonant torus exists in the phase space. Its structure can better be visualized in Poincaré section [Fig. 5(a)]. It is formed by unstable manifolds of the saddle cycle, which are closed on the stable cycle. In the absence of noise the stable cycle attracts all trajectories in the vicinity of the resonant torus. As one leaves the tongue through the curve $\mathrm{SN}$, the saddle and stable cycles merge and disappear, representing the bifurcational manifestation of phase locking breakdown.

Let us assume that the minimal distance along the torus between the saddle and stable cycles is less than half of the torus circumference. When noise is applied, fluctuations can throw the trajectory outside the region bounded by stable manifolds of the saddle cycle (SC) [far right in Fig. 5(a)], after which the trajectory returns rapidly to the stable cycle $C$ along the unstable manifold of SC. Thus an additional rotation around the torus appears, corresponding to phase slips. If the noise is very small, the slips occur rarely; and as the noise intensity grows, the slips appear more frequently. But it is clear that very strong noise will simply smear the whole dynamics. Therefore, the regularity of the new motion will depend nonmonotonically on the noise intensity. This situation is very similar to that described in Ref. [7]. An important difference in the present case, however, is that events take place on the resonant torus surface. This means that the frequency of the noise-induced oscillatory component at birth will be close to that of the synchronized oscillations (see Fig. 3), rather than close to zero as in Ref. [7]. Strictly speaking, the new timescale will be defined by the times needed to throw the trajectory from vicinity of stable cycle, and of rotation around the torus which, in terms of Ref. [8], are the
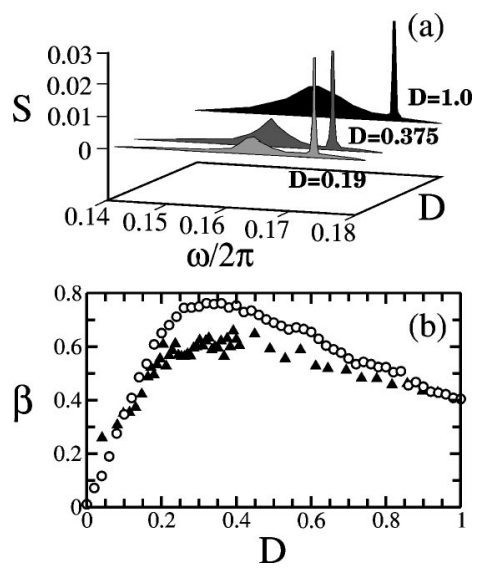

FIG. 6. Results from the electronic experiment for the case of synchronization via suppression. (a) Spectra of oscillations. (b) Regularity $\beta$ of the noise-induced motion as a function of noise intensity $D$. The notation is as in Fig. 4 .

"activation" and "excursion" times, respectively. Note that, inside the region where the resonant torus exists (below curve $B$ in Fig. 2), the noise is almost always able to induce coherent motion. An exception is the (improbable) degenerate situation that arises where distances along the torus surface between the stable and unstable cycles are exactly the same, whether moving clockwise or anticlockwise.

Now, consider the upper part of the synchronization tongue. Here, there is no torus in the phase space, and the one stable limit cycle that exists [Fig. 5(b)] attracts all trajectories from a certain vicinity. The mechanism of noiseinduced coherent motion described above then becomes impossible. The properties of the attracting cycle $C$ differ between different regions of the upper part of the tongue. Namely, between curves $T$ and $A$ the trajectories tend to $C$ while rotating along spirals, as shown in Fig. 5(b). But in the region bounded by curves $A$ and $B$ this rotation is absent. Thus if noise perturbs the system in the former case, a competition between two types of motion may be inferred, i.e., rotation along a spiral will interact with fluctuational motion. This situation is related to that described in Ref. [9], where the effect of noise on two coupled discrete maps was studied. Noise will kick the trajectory from the stable cycle evoking rotations along spirals, that is, inducing coherent oscillations. However, it is clear that the rotations themselves are also affected by fluctuations and that strong noise will just smear them. Thus, for a certain moderate noise we can expect maximally coherent motion around the stable cycle, i.e., the occurrence of CR.

To test this inference, we set $C=0.48$ and $\Omega=1.129$, close to the Neimark-Sacker bifurcation line $T$ inside the synchronization region (Fig. 2) and followed the evolution of the spectrum of oscillations with increasing noise intensity [Fig. 6(a)]. As before, noise gives rise to a new spectral peak whose width and height are controlled by noise intensity. The measured regularity $\beta$ of the new motion is plotted in Fig. $6(\mathrm{~b})$. It is interesting that for synchronization via suppression, $\beta$ also exhibits a nonmonotonic variation with $D$. Again, the analog and numerical results are in satisfactory agreement. 
In marked contrast, inside the region bounded by curves $A$ and $B$ in Fig. 2, increasing noise intensity leads only to the growth of a noisy background in the spectra, and thus no coherent motion is generated. To summarize, we have demonstrated that noise applied to a synchronized system plays a dual role. On the one hand, it disrupts synchronization. But at the same time it produces a new ordered motion whose coherence depends resonantly on the noise intensity. There is an optimal value of noise intensity which produces maximally regular biperiodic oscillations, and thus CR. The underlying CR mechanisms differ for synchronization via frequency and phase locking and via suppression of the natural dynamics. For locking, the noise induces rotations along the torus, transverse to the stable cycle lying on it. It looks like phase slips, whose frequency depends on the noise intensity.
For suppression, noise induces rotations around what is the only stable cycle in the system. The present results are interesting, not only in terms of fundamental physics, but also because they may also be relevant to a range of interdisciplinary problems, e.g., in neuroscience and biology, where synchronization phenomena occur in the presence of fluctuations.

We are grateful to D. G. Luchinsky and I. Kh. Kaufman for help with analog experiments and to the Leverhulme Trust, the Medical and the Engineering and Physical Sciences Research Councils (U.K.), the U.S. Civilian Research Development Foundation (Grant No. REC 006) and the RFBR (Grant No. 01-02-16709) for financial support.
[1] I.I. Blekhman, Synchronization in Science and Technology (ASME Press, New York, 1988); J. Guckenheimer and P. Holmes, Nonlinear Oscillations, Dynamical Systems, and Bifurcations of Vector Fields (Springer-Verlag, New York, 1986).

[2] Y. Kuramoto, Chemical Oscillations, Waves and Turbulence (Springer, Berlin, 1984); C. Terquem et al., Astrophys. J. 502, 788 (1998); Stephen A. Drake et al., Astron. J. 115, 2122 (1998); D. Lai et al., Astrophys. J. 423, 344 (1994); H.J. Freund, Physiol. Rev. 63, 387 (1983); C. Schäfer, M.G. Rosenblum, J. Kurths, and H.H. Abel, Nature (London) 392(6673), 239 (1998); A. Stefanovska, H. Haken, P.V.E. McClintock, M. Hožič, F. Bajrović, and S. Ribarič, Phys. Rev. Lett. 85, 4831 (2000).

[3] R. L. Stratonovich, Topics in Theory of Random Noise (Gordon and Breach, New York, 1967), Vol. 2.

[4] A.S. Pikovsky, M.G. Rosenblum, and J. Kurths, Int. J. Bifurcation Chaos Appl. Sci. Eng. 10, 2291 (2000); A.B. Neiman, A. Silchenko, V.S. Anishchenko, and L. Schimansky-Geier, Phys. Rev. E 58, 7118 (1998).

[5] J.M.G. Vilar and J.M. Rubi, Phys. Rev. Lett. 86, 950 (2001).

[6] R. Benzi, A. Suera, and A. Vulpiani, J. Phys. A 14, L453 (1981); P. Jung, Phys. Rep. 243, 175 (1993); M.I. Dykman, D.G. Luchinsky, R. Mannella, P.V.E. McClintock, N.D. Stein, and N.G. Stocks, Nuovo Cimento D 17, 661 (1995); J.M.G. Vilar and J.M. Rubi, Phys. Rev. Lett. 78, 2886 (1997); V.S.
Anishchenko, A.B. Neiman, F. Moss, and L. SchimanskyGeier, Phys. Usp. 42, 7 (1999).

[7] H. Gang, T. Ditzinger, C.Z. Ning, and H. Haken, Phys. Rev. Lett. 71, 807 (1993); W.-J. Rappel and S.H. Strogatz, Phys. Rev. E 50, 3249 (1994); D. Sigeti and W. Horsthemke, J. Stat. Phys. 54, 1217 (1989).

[8] A.S. Pikovsky and J. Kurths, Phys. Rev. Lett. 78, 775 (1997).

[9] A. Neiman, P.I. Saparin, and L. Stone, Phys. Rev. E 56, 270 (1997).

[10] D.E. Postnov, S.K. Han, T.G. Yim, and O.V. Sosnovtseva, Phys. Rev. E 59, R3791 (1999); S.K. Han, T.G. Yim, D.E. Postnov, and O.V. Sosnovtseva, Phys. Rev. Lett. 83, 1771 (1999).

[11] D. Sigeti and W. Horsthemke, J. Stat. Phys. 54, 1217 (1989); M. Qian et al., Phys. Rev. E 62, 6469 (2000).

[12] D.G. Luchinsky, P.V.E. McClintock, and M.I. Dykman, Rep. Prog. Phys. 61, 889 (1998).

[13] The correlation time of the Gaussian noise was much smaller than the relaxation time of the circuit, so that it could therefore [12] be considered as white.

[14] V.I. Arnold, Geometrical Methods in the Theory of Ordinary Differential Equations (Springer, New York, 1983).

[15] To compare the results of the analog and digital experiments, we renormalized the frequency and noise intensity to dimensionless units compatible with Eq. (1). 